\title{
Mechanical metamaterials with star-shaped pores exhibiting negative and zero Poisson's ratio
}

\author{
Luke Mizzi $^{\text {a }}$, E.M. Mahdi ${ }^{\text {b }}$, Kirill Titov ${ }^{\text {b }}$, Ruben Gatt ${ }^{\text {a }}$, Daphne Attard ${ }^{\text {a }}$, Kenneth E. Evans ${ }^{\text {c }}$, \\ Joseph N. Grima ${ }^{\mathrm{a}, \mathrm{d}, *}$, Jin-Chong Tan ${ }^{\mathrm{b}, * *}$ \\ ${ }^{a}$ Metamaterials Unit, Faculty of Science, University of Malta, Msida MSD 2080, Malta \\ ${ }^{\mathrm{b}}$ Multifunctional Materials \& Composites (MMC) Laboratory, Department of Engineering Science, University of Oxford, Parks Road, Oxford OX13PJ, United Kingdom \\ c Department of Engineering, University of Exeter, North Park Road, Exeter EX4 4QF, United Kingdom \\ d Department of Chemistry, Faculty of Science, University of Malta, Msida MSD 2080, Malta
}

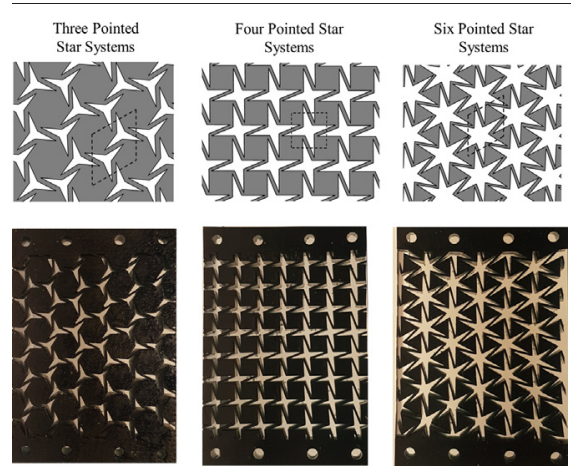

\section{A R T I C L E I N F O}

\section{Article history:}

Received 18 December 2017

Received in revised form 2 February 2018

Accepted 17 February 2018

Available online 24 February 2018

\section{Keywords:}

Mechanical metamaterials

Auxetics

Finite element analysis

3D printing

Perforated systems

\section{A B S T R A C T}

The term "mechanical metamaterials" encompasses a wide range of systems whose anomalous mechanical properties arise primarily from their structure rather than composition. This unique characteristic gives them an edge over many conventional natural or readily available materials and makes them well-suited for a variety of applications where tailor-made mechanical properties are required. In this study, we present a new class of mechanical metamaterials featuring various star-shaped perforations, which have the potential to exhibit auxetic or zero Poisson's ratio $(v)$ properties. Using finite element modelling in conjunction with experimental measurements on 3D printed prototypes, we demonstrate that these star-shaped porous systems possess the potential to retain their unusual mechanical properties up to tensile strains exceeding 15\%. By virtue of these exceptional properties, the proposed concept could be applied for engineering numerous potential applications in a wide range of fields. (C) 2018 Published by Elsevier Ltd.

\footnotetext{
* Correspondence to: J. N. Grima, Metamaterials Unit, Faculty of Science, University of Malta, Msida MSD 2080, Malta.

** Corresponding author.

E-mail addresses: joseph.grima@um.edu.mt (J.N. Grima), jin-chong.tan@eng.ox.ac.uk (J.-C. Tan)
}

\section{Introduction}

Mechanical metamaterials are systems that derive their anomalous mechanical response to external stimuli primarily from their geometric structure rather than from their intrinsic material composition [1]. In recent years, there has been a great deal of interest in these systems 
amongst scientists and engineers since they offer an unconventional route to the production of materials with tailor-made mechanical properties, which might otherwise be difficult to achieve simply by adopting natural or readily available isotropic materials. One of the most wellknown classes of mechanical metamaterials is that of auxetic systems. Auxetics [2] are systems which possess the counter intuitive mechanical property of exhibiting a negative Poisson's ratio, i.e. they expand in a transverse direction upon the application of a uniaxial tensile strain. This property, which stems from the geometry and deformation mechanism of the system, has been reported in several naturally occurring and synthetic systems, ranging from the nano- to the macro-scale, such as zeolites [3], metal-organic frameworks [4], graphene [5], carbon nanotubes [6], foams [7,8], fibres [9], polymers [10], biological [11] and perforated systems [12]. Auxetic materials have also been shown to exhibit numerous advantageous characteristics which are not typically observed in conventional materials, such as the ability to undergo synclastic curvature [13], increased indentation resistance [14] and sound dampening properties $[15,16]$. These features make them especially suited for a number of niche applications in a wide variety of fields, encompassing biomedicine to electronic devices [16-21].
Despite the vast potential of auxetic mechanical metamaterials, which are superior to naturally occurring auxetics due to their increased versatility, one of the main challenges faced by researchers in implementing these systems for real world applications and devices is optimization of the production method. The procedure of making an auxetic metamaterial typically involves a multi-step manufacturing process, which includes the attainment of a specific geometrical configuration to achieve the auxetic effect, ideally with a high degree of precision via production techniques such as 3D printing [22-25], laser lithography [26], molding [27] and perforation methods [12,20,21,2831]. The suitability of a manufacturing process relies heavily on the type of geometric features that need to be introduced, which could present a challenge, especially if the auxetic properties are required at the micro- or nano-level. For example, standard low resolution 3D printers might not be ideal to produce auxetic metamaterials based on rotating rigid unit and re-entrant geometries through slits [30] due to the minute thickness of the pores. On the other hand, such printers may be considered to be more suited for the fabrication of highly porous metamaterials. Therefore, in their quest to manufacture new and improved mechanical metamaterials, scientists and engineers often must either a) i)

Three-Pointed Star System

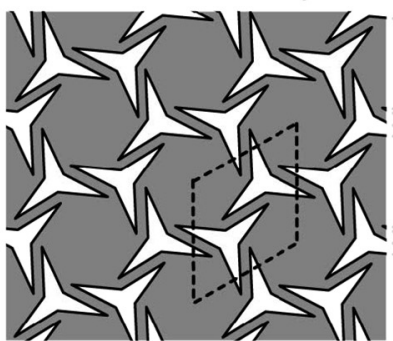

ii)

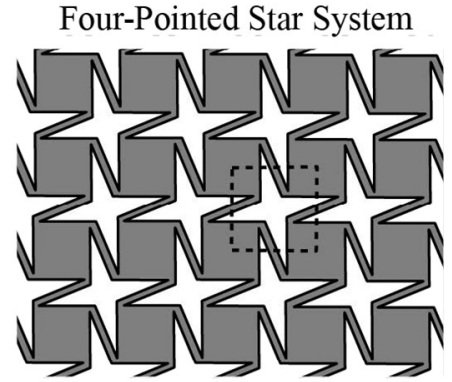

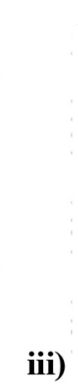
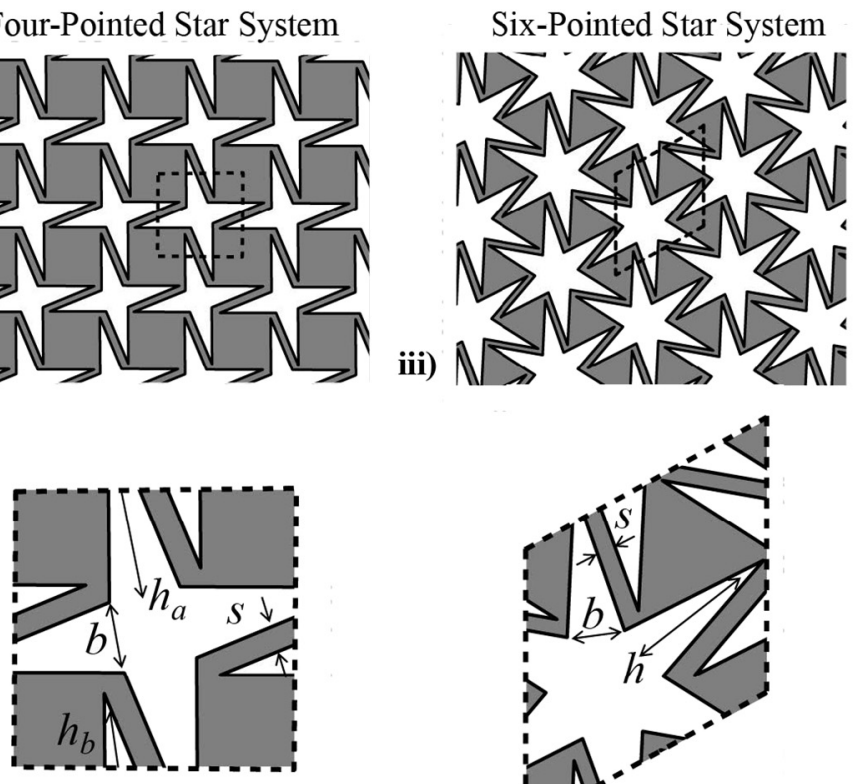

ii)

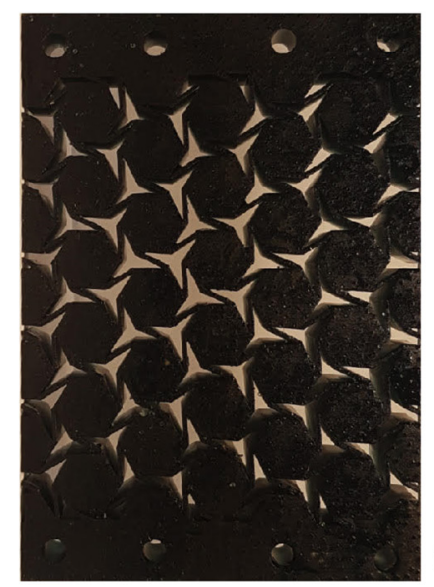

ii)

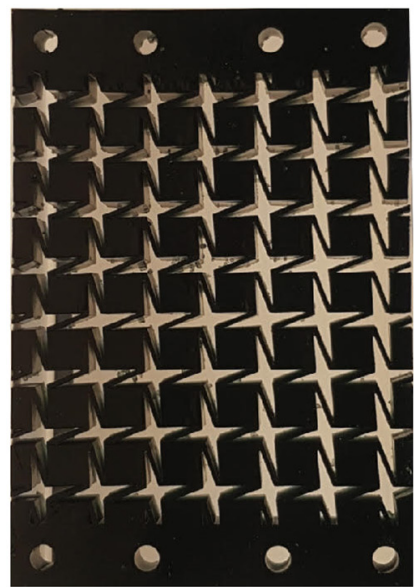

iii)
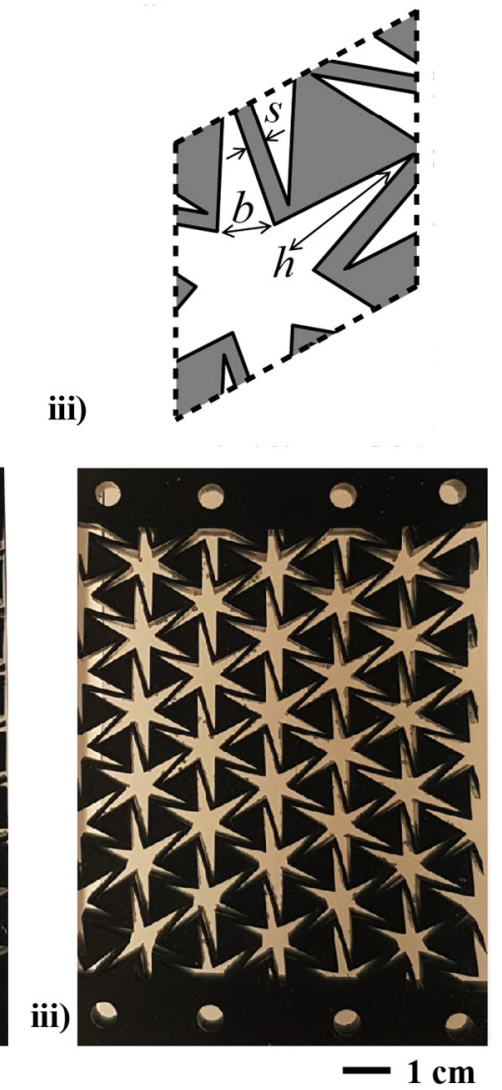

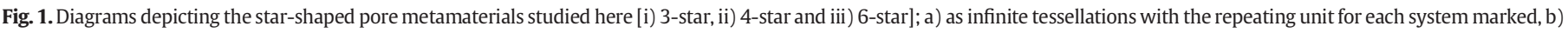

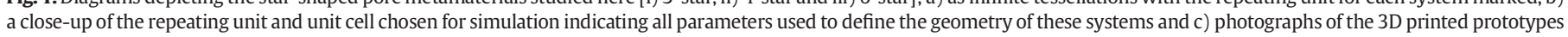
of these geometries which were used in the experiments. 
improve the manufacturing technique, or, come up with alternative designs that are less problematic to implement but still able to produce the required mechanical response. Thus it is envisaged that by having a large pool of geometries with a wide spectrum of mechanical properties from which to 'pick and choose', the increased geometric versatility could allow one to bypass any complications which may arise during the manufacturing process, and thus, in the future any mechanical metamaterials with a specific tailored set of mechanical properties could be produced using a standard fabrication technique.

Considering the aforementioned challenges, in this work we are proposing a new class of perforated auxetic mechanical metamaterials with the potential to exhibit Poisson's ratios within -1 to 0 . These systems, possess $n$-pointed symmetric star-shaped pores, where $n$ may be equal to 3, 4 or 6, are arranged as shown in Fig. 1. The on-axis mechanical properties of these systems were investigated using a finite element (FE) modelling approach, which was supported by experimental measurements performed on 3D printed prototypes manufactured using a Formlabs Form 2 3D stereolithography laser printer (with a print resolution of $\sim 50 \mu \mathrm{m})$.

\section{Methodology}

The finite element (FE) analyses were conducted using the ANSYS13 software (ANSYS Inc). As shown in Fig. 1b, each of these systems are defined by a distinct set of parameters; namely $h$ and $b$, the height and base length of the triangles that make up the star-shaped perforation and $s$, which is the separation distance between the two adjacent star perforations. To obtain a clear picture of the potential mechanical properties of these systems, FE simulations were conducted on a wide range of structures by systematically varying the values of these parameters. More precisely, for $n=3,4$ and 6, the parameter $h$ was set to values from 1 to 6 in steps of 1 , while $s$ was set to values ranging from 0.1 to 0.6 in steps of 0.05 . The parameter $b$ was kept constant at 1 for all the structures.

The systems were modelled using the PLANE183 element, a plane stress, higher order 2D 8-node or 6-node element with quadratic displacement behaviour. Following the numerical convergence testing of the FE models, a minimum mesh size of 0.02 was used for all systems, which is equivalent to $1 / 5$ of the minimum $s$ dimension of the systems being investigated here. The linear material properties of the cured Tough Resin (a commercial-grade hyperelastic polymer developed by Formlabs Inc.) were used to simulate these systems, i.e. a Poisson's ratio of 0.49 and a Young's modulus of $1.68 \mathrm{GPa}$ (see S1 in Supplementary Information (SI) for details), and to maximize computational efficiency, each system was modelled as a representative unit cell (see Fig. 1b) with periodic boundary conditions and solved linearly. These periodic boundary conditions were enacted through the use of constraint equations on the nodes at the edges of the system. These constraint equations operate under the assumption that in order for a system to be periodic the nodes on opposing boundaries of the representative unit cell must deform in an identical manner. In addition, in order to eliminate any artificial constraints on the systems which could influence their deformation behaviour, the systems were constrained from two nodes on opposing edges of the representative unit cell only and loading was simulated via the application of a uniaxial tensile force on the nodes at the edges of the unit cell in the $x$ - and $y$-directions separately. This results in a system whose deformation is not governed by artificial edge effects and thus represents the deformation of a system with a very large number of repeating units. The on-axis Poisson's ratios and Young's moduli of the systems were calculated from the strains on the nodes at the edges of the unit cells, which in turn, were calculated from the displacements of the nodes induced by loading. The effective Young's modulus was calculated as a percentage of the Young's modulus of the system in comparison to the material Young's modulus.
These linear simulations were conducted mainly in order to obtain a clear insight of the small strain mechanical properties of these systems. In order to obtain a more complete picture of the deformation mechanism/s governing the mechanical behaviour of these systems, as well as to investigate their high strain properties, further studies were also conducted on one example of each of the three systems investigated here using non-linear Finite Element simulations and experimental testing. The experimental prototypes of the three models, one of each for $n=3,4$ and 6 were produced using the Tough Resin [32] using the Formlabs 3D Printer (see Fig. 1c). The three systems possessed the following parameters:

\section{a. Three-Star Perforated System (Fig. 1c(i))}

Gauge Length $=106 \mathrm{~mm}$, Number of Horizontal Repeating Units = 6 , Number of Vertical Repeating Units $=6, h=8 \mathrm{~mm}, b=3 \mathrm{~mm}, s$ $=1 \mathrm{~mm}$, Depth $=10 \mathrm{~mm}$

b. Four-Star Perforated System (Fig. 1c(ii))

Gauge Length $=107 \mathrm{~mm}$, Number of Horizontal Repeating Units = 7, Number of Vertical Repeating Units $=8, h=10 \mathrm{~mm}, b=3 \mathrm{~mm}$, $s=1 \mathrm{~mm}$, Depth $=10 \mathrm{~mm}$

c. Six-Star Perforated System (Fig. 1c(iii))

Gauge Length $=104 \mathrm{~mm}$, Number of Horizontal Repeating Units = 6 , Number of Vertical Repeating Units $=6, h=10 \mathrm{~mm}, b=3 \mathrm{~mm}$, $s=1 \mathrm{~mm}$, Depth $=10 \mathrm{~mm}$.

These star perforation parameters were chosen on the basis of the results of the linear simulations, while the final sample size and number of repeating units were chosen with the explicit aim of producing a model with the maximum permissible number of repeating units (to minimize the influence of edge effects on the repeating units in the center of the sample from which the extension measurements were taken). At the same time, we have ensured that the dimensions of the repeating units are not too small that defects arising from the 3D printer resolution will play a significant role in the deformation of the final sample.

These 3D printed models were stretched using a Testometric tensile loading machine at a rate of $1.5 \mathrm{~mm} / \mathrm{min}$. Markers were drawn on the repeating unit cells at the centermost region of each model and a video extensometer (Messphysik) was used to measure the displacement of these markers during loading. These displacements were then averaged and used to calculate the instantaneous and engineering strains, from which the Poisson's ratio was then derived.

In order to allow for a proper comparison with the FE results, two additional sets of non-linear simulations were conducted for each of these systems. For both these sets of simulations, the non-linear material properties of Tough Resin were used. These material properties were found by testing multiple dog-bone shaped samples in a tensile loading machine and fitting the resultant stress-strain graph with 5-Parameter Mooney-Rivlin Hyper-elastic model (see S1 in the Supplementary Information for further details). The first set of non-linear simulations was run using the same periodic boundary conditions as those employed for the linear simulations while for the second set, identical models corresponding to each of the three 3D printed prototypes were designed and simulated for the same loading conditions in the tensile loading machine. This entailed fixing the nodes in the lower part of the system in the $x$ - and $y$-directions and applying a strain in the $y$-direction and fixing the displacement in the $x$-direction of the nodes in the upper part of the system. The Poisson's ratio of each system was also calculated from the centermost region of the model; identical to the method employed for the experimental prototypes. The final plots for the Poisson's ratio of all the non-linear simulations and experimental tests were plotted as the instantaneous Poisson's ratio [33] against engineering strain (see S1 in Supplementary Information for more details). 
Three Pointed Star Systems

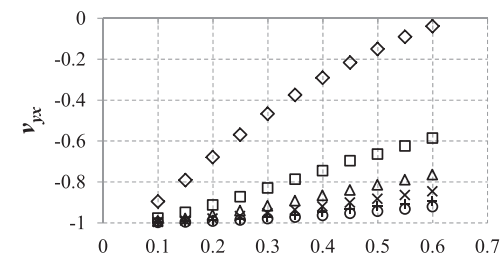

a) i)

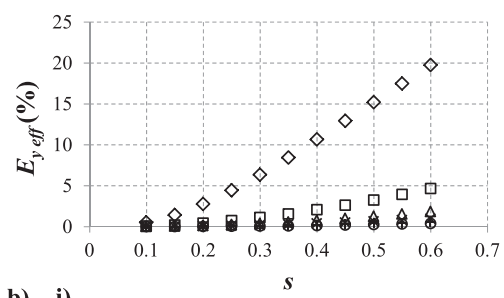

b) i)
Four Pointed Star Systems
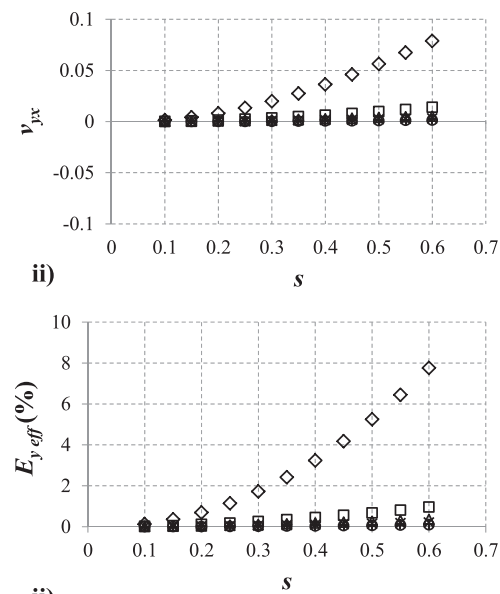

ii)
Six Pointed Star Systems
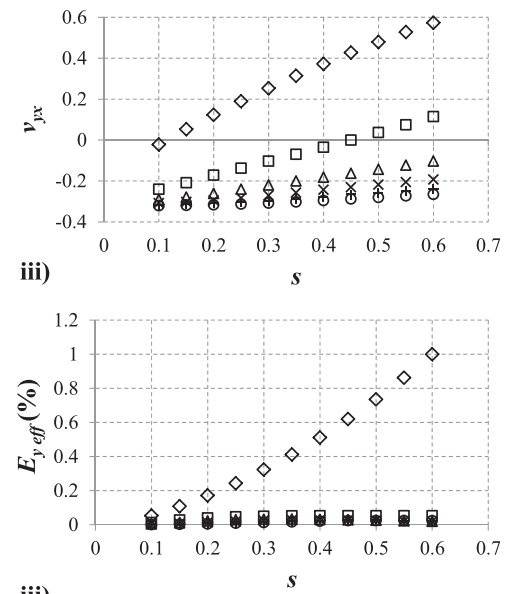

iii)
$\diamond h=1$
$h=2$
$\triangle h=3$
$\times h=4$
$+h=5$
○ $h=6$

Fig. 2. Plots showing the simulated on-axis a) Poisson's ratios, $\nu_{y x}$ and b) effective Young's moduli, $E_{y}$ eff (as a percentage of the material Young's modulus, $E_{\text {mat }}$ ) for the i) 3-star, ii) 4-star and iii) 6 -star pore shaped systems for the application of a uniaxial load in the $y$-direction. The results for stretching in the $x$-direction are nearly identical to these and can be found in Fig. S5 of the Supplementary Information.

\section{Results and discussion}

As shown in Fig. 2 the three-pointed star and six-pointed star systems are predicted by the linear simulations to potentially exhibit varying extents of auxetic behaviour, while the majority of the four-pointed star systems show a Poisson's ratio of approximately zero. It can be seen that almost all systems are predicted to exhibit a relatively low effective Young's modulus (less than $1 \%$ of the Young's modulus of the tough resin material ( $\sim 17 \mathrm{GPa}$ ) for most systems being studied). This, coupled with the fact that the material Poisson's ratio was 0.49 (nearly incompressible), confirms that the macroscopic mechanical properties of the system is expected to be governed primarily by the geometrical structure of the system rather than by uniform deformation of the polymeric resin material. In fact, an inspection of the systems suggest that uniaxial loading resulted in changes in the geometric features of the systems, whose changes are concordant with the Poisson's ratio of the respective systems as explained below. Moreover, all systems exhibit nearly identical on-axis mechanical properties for tensile loading in both the $x$ - and $y$-directions (see Fig. 2 vs. Fig. S5), as expected from the symmetry of the respective systems.

As presented in Figs. 4, 5 and 8 and the videos in the Supplementary Information (ANIM01-09), the star-shaped systems are deforming via a mechanism which may be roughly described in terms of bending of the flexible ligament-like connectors and/or rotation of the polygonal blocks of material. Given their structural symmetry and deformation behaviour, these systems may be likened to chiral honeycombs [34-42]; namely the hexachiral, tetrachiral and trichiral honeycombs for the three-pointed star, the four-pointed star and the six-pointed star pored systems, respectively. Typical chiral honeycombs [34] reported in literature are usually made up of circular nodes that are connected to each other through tangentially attached ligaments. However, despite the similarities, the systems proposed here differ from these geometries in several key aspects, with the most obvious differences being the polygonal shape of the 'chiral block', as opposed to a circular node, and the fact that the 'ligaments' need not necessarily be tangentially oriented with respect to the center of the chiral units. The latter point means that the angle between the ligaments and the center of the chiral node could potentially be designed to be lower than $90^{\circ}$. These structural differences are expected to increase the strain range over which auxetic behaviour is observed for tensile loading compared with typical chiral geometries, since these systems are in an initial geometric state that is more distant from the theoretical tensile strain limit of the chiral honeycomb mechanism (see Fig. 3). Similarly, the systems may also be designed to exhibit a superior capacity for compressive
Partially Closed State

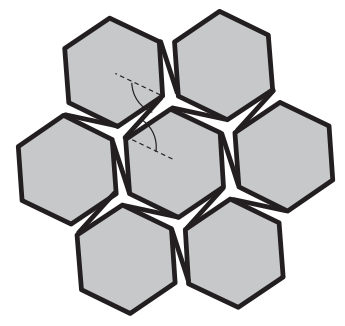

Tangentially Attached

Ligaments

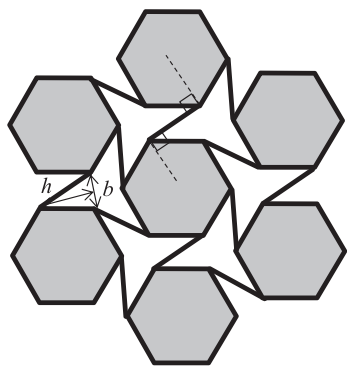

Fully Opened State

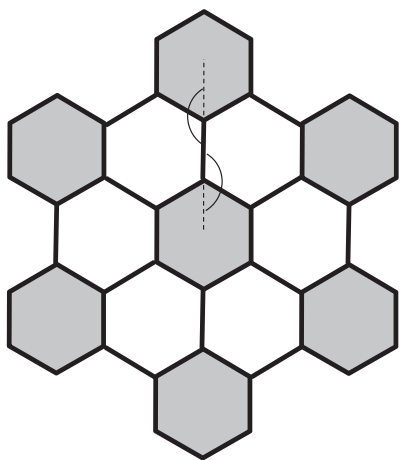

Fig. 3. Diagrams showing the partially closed, tangential orientation and theoretically fully opened state of one of the three-pointed star-shaped perforation system studied here. 
strains by producing systems with angles greater than $90^{\circ}$. The size of this angle is governed by the relationship between the perforation parameters $h$ and $b$ and may be defined as $2 \operatorname{atan}(b / 2 h)+((N-2) \pi) /$ $(2 N)$ where $N$ denotes the number of sides of the polygonal units. This means that as the $h / b$ ratio increases, the size of the angle will approach $((N-2) \pi) /(2 N)$ and the star-shaped perforation will start to resemble a slit.

Here it must be noted that the tangentially attached ligament equivalents of these systems corresponding to typical circular node chiral honeycombs are analogous to geometries where the star-shaped perforations almost lose their convex angle and become polygonally shaped, i.e. a triangle, a square and a hexagon in the case of the three, four and six-pointed star pores, respectively. This corresponds to systems where the $b / h$ ratio is equivalent to $2(\tan (\pi / 6)), 2(\tan (\pi / 4))$ and 2 $(\tan (\pi / 3))$ respectively.

As one may observe from the FE predictions of the linear simulations in Fig. 2, the three-pointed star perforation systems appear to show the greatest potential for exhibiting auxetic behaviour, with the Poisson's ratio of these systems reaching its most negative point, a value of -1 , when the separation value, $s$, is at its lowest value. This Poisson's ratio corresponds to that predicted by analytical models for hexachiral honeycombs. In fact, as one can observe from ANIM01, ANIM04 and ANIM07, at low $s$ values, these systems deform in a manner which is analogous to the chiral mechanism, i.e. rotation of central blocks and flexural deformation of ligaments in a manner where the rotational symmetry of the building-block is preserved to a certain extent $[34,36]$. This was to be expected since at small values of $s$, the threestar perforated systems studied here would be equivalent to hexachiral systems with slender ligaments. This assertion is also supported by the fact that the systems possessing the most negative Poisson's ratios are those with the lowest stiffness values and lowest $s$ values, i.e. the systems that most effectively mimic the hexachiral mechanism and show the greatest conformation to analytical predictions for this model. On the other hand, systems with larger separations will possess thicker ligaments and, should the ligaments be sufficiently thick, other deformation mechanisms such as shear deformation of the ligaments could be relevant [43], resulting in deviations from the "pure" hexachiral mechanism. It should be pointed out that besides $s$, the magnitude of the parameters $h$ and $b$ is also a determinant of ligament slenderness since, while $s$ defines the thickness of the ligament, these parameters define the length of the ligament. In fact, as shown in Fig. 2, as the $h / b$ ratio decreases, the systems become increasingly less negative because of the increase in ligament thickness. The relationship between these parameters with the length to thickness ratio of the formed ligament may be quantified as follows: length/thickness $=\left(h^{2}+b^{2} / 4\right)^{1 / 2} / s$, and the greater the value of this ratio, the greater the conformity of the system's deformation behaviour to that of the hexachiral mechanism.

The potential of the three-pointed star perforated systems to exhibit auxetic behaviour is also clearly confirmed by the results obtained from the experimental tensile loading of the 3D printed prototypes and nonlinear FE simulations. As shown in Fig. 4, the experimental prototype of the three pointed star-shaped pore system possesses an initial negative Poisson's ratio of approximately -0.5 , with the system becoming slightly more auxetic on increasing elongation, up to a strain of ca. $17 \%$. This trend is mirrored in the non-linear FE simulation of the equivalent model using the same boundary conditions, albeit at a slightly more negative Poisson's ratio. A similar trend, with an even more negative Poisson's ratio, is also observed for the non-linear simulation conducted using periodic boundary conditions, i.e. similar to the linear simulations. These small differences in the magnitude of the Poisson's ratio may be attributed mainly to edge/boundary effects and imperfections. In the system with periodic boundary conditions, there are no boundary effects such as clamping or free edges (which are present in the finite system simulation and experimental model) and thus the system is able to deform freely. However, in the corresponding finite systems, the polygonal nodes near the top and bottom regions of the

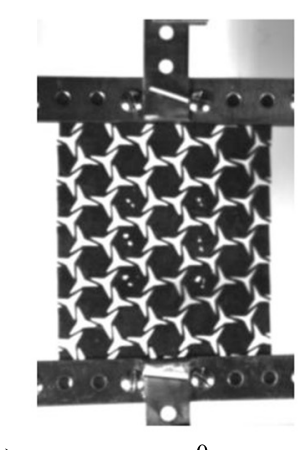

a)

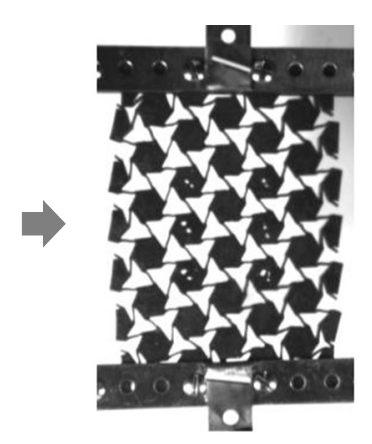

$\varepsilon_{y}=0.14$
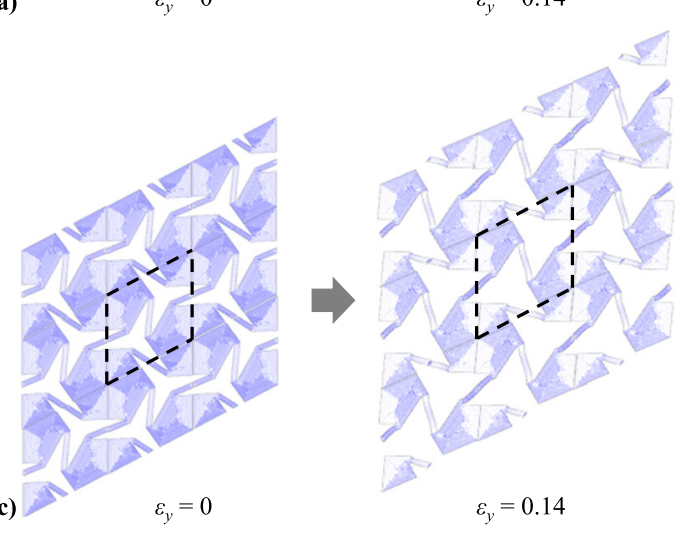

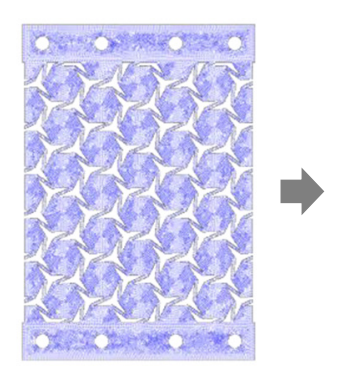

b)

$$
\varepsilon_{y}=0
$$

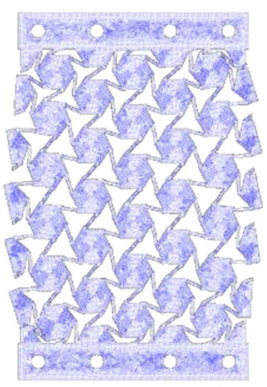

$\varepsilon_{y}=0.14$

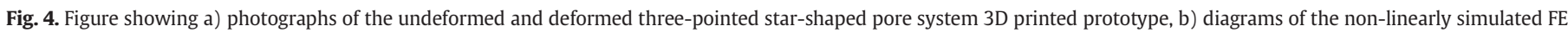

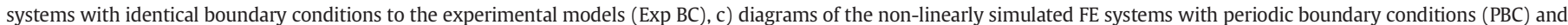

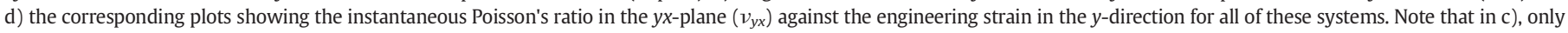

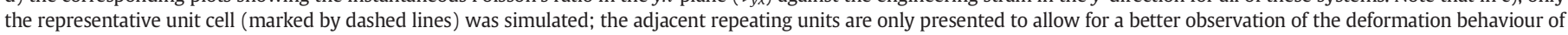
this system. 
sample are significantly more constrained than those at the central region of the system, which creates a deformation gradient throughout the sample, i.e. the topmost and bottommost nodes deform significantly less than the centermost nodes, hence the bulging out at the central regions which may be observed in Fig. 3b, c. In theory, if the sample contains a sufficient number of repeating unit cells, the central nodes should be almost completely unaffected by these edge effects and thus the deformation of the finite systems, and hence the Poisson's ratio, would be equal to that of the system modelled using periodic boundary conditions. However, in practice, this is extremely difficult to achieve and thus some discrepancies are always expected.

There is also a slight difference between the Poisson's ratio of the experimental prototype and the finite non-linear FE simulation. This difference is probably due to slight imperfections in the 3D printing process which resulted in some ligaments having unequal stiffnesses, which in turn results in uneven deformation throughout the system which affects the overall Poisson's ratio. However, despite these differences, as one can observe from Fig. 4 and ANIM01, ANIM04 and ANIM07, all three systems still deform predominantly by roughly the same deformation mechanism, i.e. rotation of the polygonal chiral nodes and flexure of ligaments.

In the case of the four- and six-pointed perforated star systems, the deformations are more complex. For example, as one can observe from Fig. 5, in the case of the four-pointed star systems, small values of $s$ results in geometries where the system may be likened to a tetrachiral system with square-shaped nodes interconnected by four slender ligaments. However, in this case the horizontally inclined ligaments undergo a higher degree of flexural deformation than their vertically inclined counterparts, meaning the rotational symmetry of order four is not being preserved. This effect has been previously observed in tetrachiral honeycombs $[36,39]$, and was to be expected since the system lacks the required symmetry and/or structural constraints to preserve the four-fold rotational symmetry of the sub-units. Unequal flexure arises since one set of ligaments has a higher component of force in the loading direction than the other. This leads to the system experiencing significant shear deformation (see Figs. 5c, 6 and ANIM08), which if not restricted, would result in a Poisson's ratio of zero, as predicted by the FE simulations reported in Fig. 2 .

The results obtained from the experimental loading experiment and non-linear FE simulations at high strains of the four-pointed starshaped pore system confirm this hypothesis. In fact, in the non-linear FE simulation where the system was loaded under conditions which impose no artificial constraints besides those governing its periodicity, the system showed significant shear deformation upon uniaxial loading resulting in an initial Poisson's ratio of zero which very slowly becomes positive over significantly large strains (see Fig. 5), in accordance with earlier theoretical predictions [36,39]. On the other hand, during experimental testing of the 3D printed model and the equivalent non-linear FE model, the system was clamped from the top and bottom parts while being stretched, resulting in an initially experimentally measured Poisson's ratio of -0.1 , since this type of loading discourages shearing deformations as the extremities of the model have to remain normal to the clamps throughout testing. However, a degree of shear deformation is still evident at higher tensile strains, which suggests that the clamping method does not fully inhibit this mode of deformation (see Fig. $6 b(i)$ ). Since shear deformation is inhibited, this system deforms through a mixture of unequal flexural deformation (which results in shearing) and rotation of the chiral nodes (see Fig. 6a), with the latter mechanism being responsible for the overall negative Poisson's ratio. As tensile strain increases, the Poisson's ratio gradually becomes even more negative as the rotation of nodes becomes the more predominant form of deformation. This change in deformation mechanism, which may be easily observed from the stress contour plots presented in Fig. $6 \mathrm{~b}$, highlights the significant role of boundary conditions on the a)
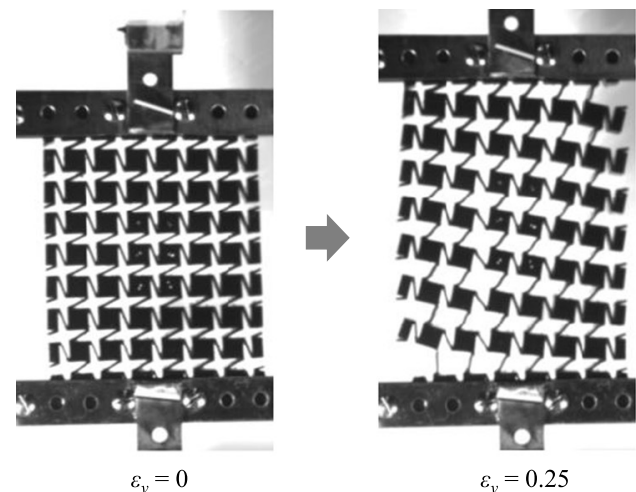

$\varepsilon_{y}=0.25$
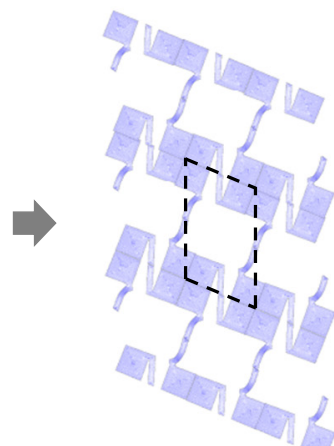

$\varepsilon_{y}=0.25$

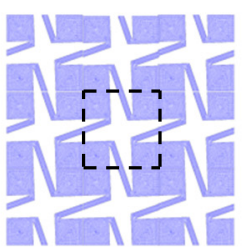

$$
\varepsilon_{y}=0
$$

b)

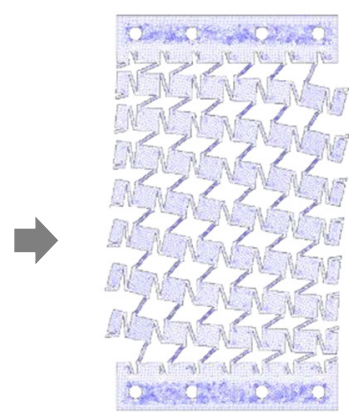

$\varepsilon_{y}=0.25$

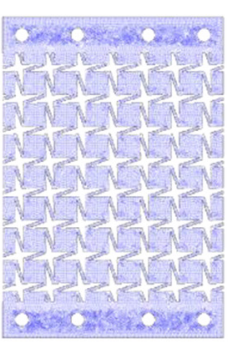

$$
\varepsilon_{y}=0
$$

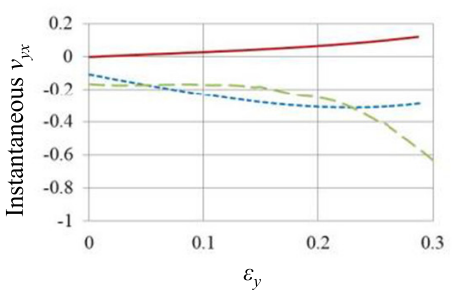

Experimental $---\mathrm{FEA}(\operatorname{Exp} \mathrm{BC})$ FEA (PBC)

d)

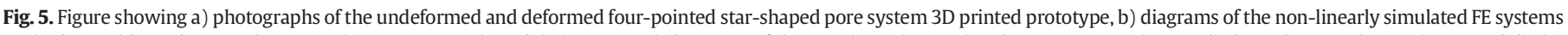

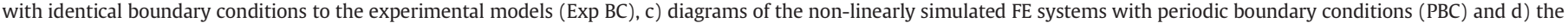

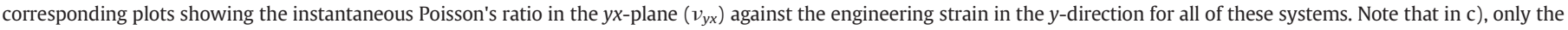

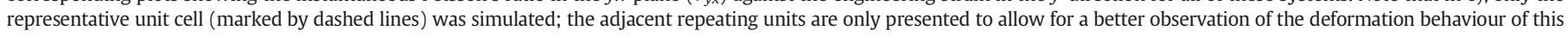
system. 


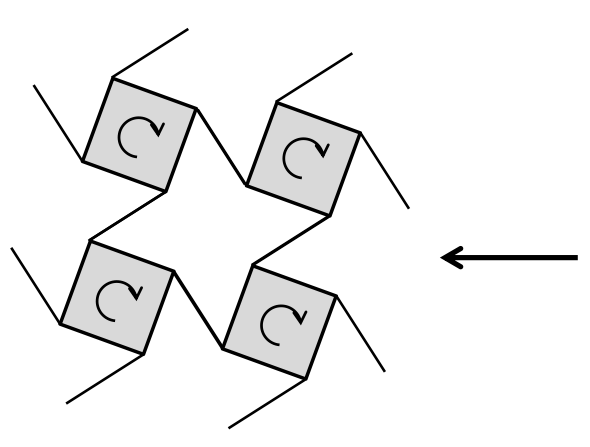

a) Uniform Rotation Deformation

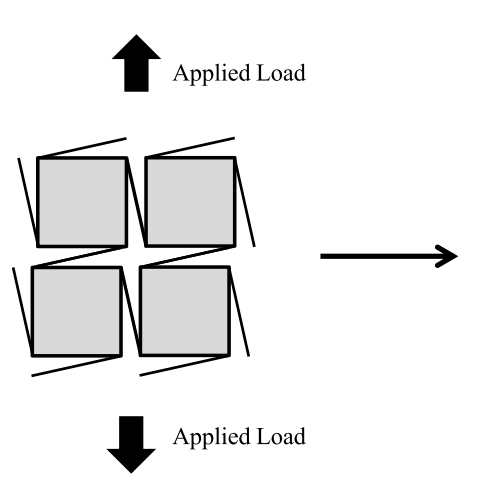

Initial Form

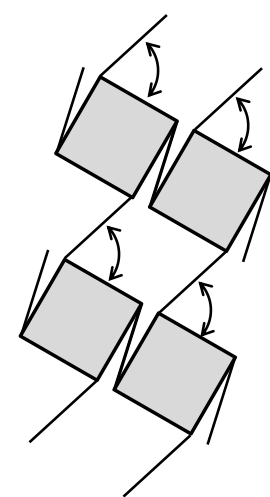

Shear-Directed Deformation

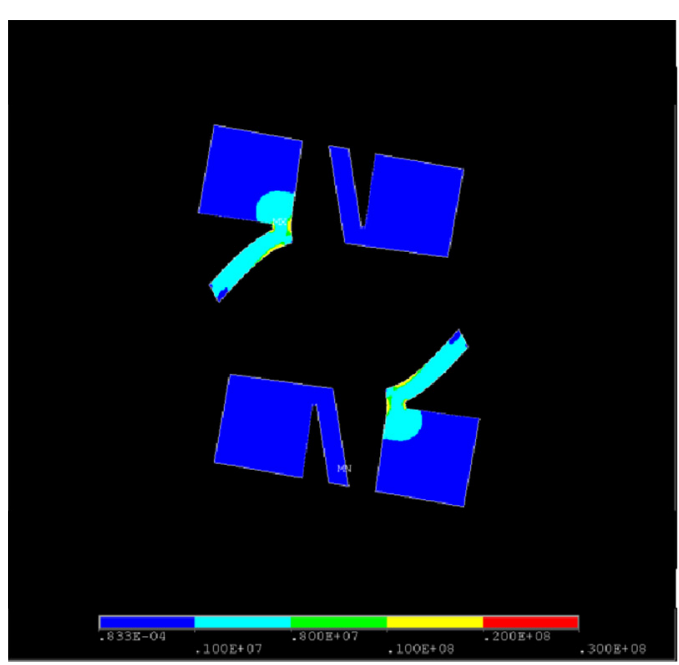

b) i)

Non-linear FE using PBCs

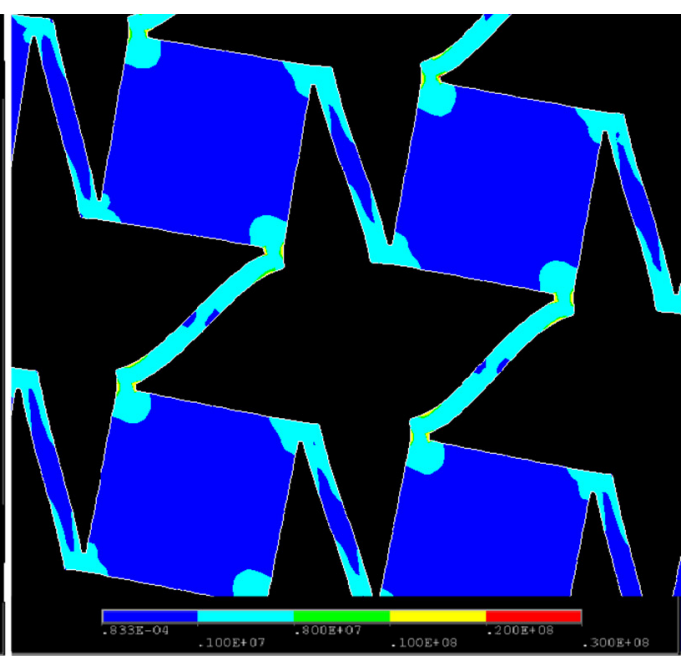

ii) Non-linear FE using the Tensile Loading BCs

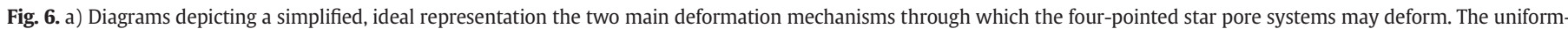

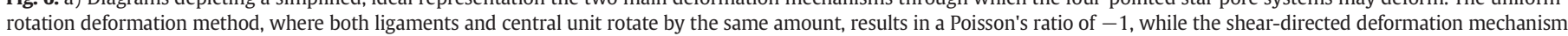

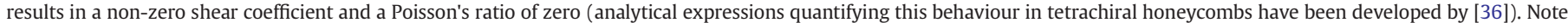

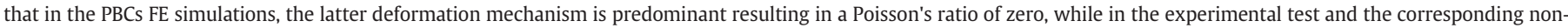

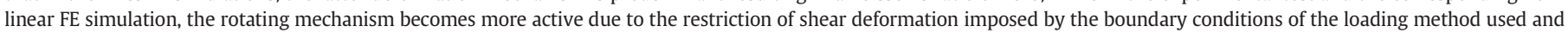

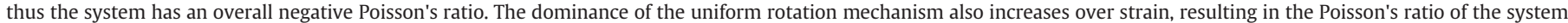

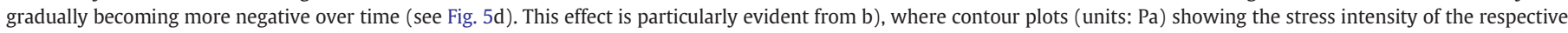

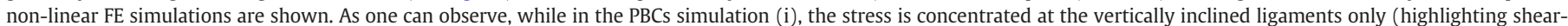

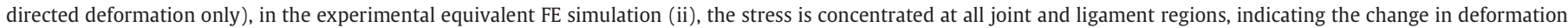
mechanism observed between these two systems.

mechanical properties and deformation behaviour of four-star systems, where unlike the three-pointed star systems, the deformation may be completely controlled by the method through which the system is loaded.

In the case of the six-pointed star system, both non-linear simulations and tests on the experimental prototype showed that this system exhibits a negative Poisson's ratio. Similarly to the four-pointed star perforated system, ligament deformation is not uniform with the three sets of ligaments surrounding each chiral node deforming to different extents. In this case, the resulting deformation is one that can be described primarily in terms of rotations and flexure of the ligament-like units, with influences of elements of re-entrancy, which cooperatively result in a significantly negative Poisson's ratio which is retained over a considerable tensile strain range. These two mechanisms (shown in Fig. 7) are both occurring concurrently, however unlike in the four-pointed star system where the two competing mechanisms possess zero and negative Poisson's ratios, in this case both mechanisms result in auxetic behaviour, hence the system's overall negative Poisson's ratio.

As shown in Fig. 8 both non-linear FE simulations and the experimental results show almost identical behaviour, with the Poisson's ratio starting from a negative value, which decreases gradually over a strain range of $\sim 24 \%$. As expected, the non-linear FE simulation with periodic boundary conditions predicts the lowest Poisson's ratio (initial $\nu_{y x}=-0.28$ ). This is followed by the non-linear FE simulation with clamped upper and lower boundaries (initial $\nu_{y x}=-0.21$ ) and the experimental prototype (initial $\nu_{y x}=-0.16$ ). These discrepancies are probably due to the same reasons explained previously for the threestar system, since as one may observe from Fig. 8 and ANIM03, ANIM06 and ANIM09, all three systems appear to be deforming basically in almost the same manner.

Another interesting finding to highlight for all three systems studied here is that in extreme cases where the separation between perforations, $s$, and the $h / b$ ratio are very high and very low respectively, one would expect the Poisson's ratio and Young's modulus of the system to tend towards that of the bulk material since the effectiveness of the respective mechanisms responsible for auxeticity/zero Poisson's ratio to function would be significantly reduced. This appears to be the case for the three- and four-pointed star systems; however, in the case of the six-pointed star perforated systems, the Poisson's ratio becomes even more positive than that of the original material $(+0.49)$ as 


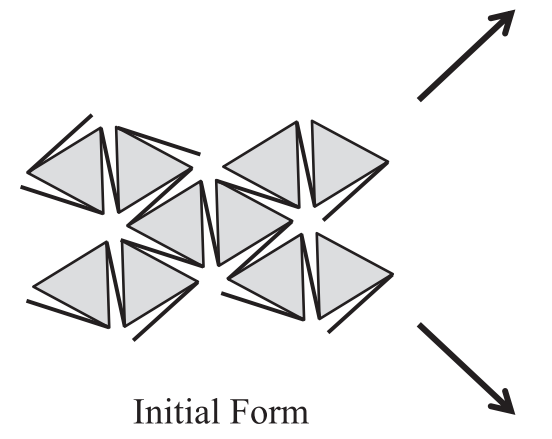

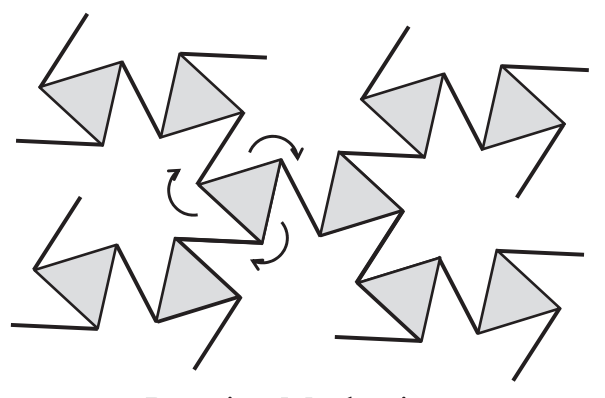

Rotating Mechanism

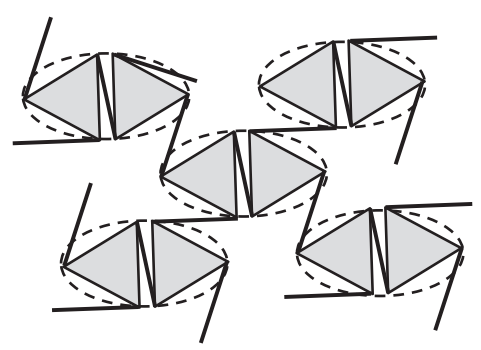

Re-entrant Mechanism

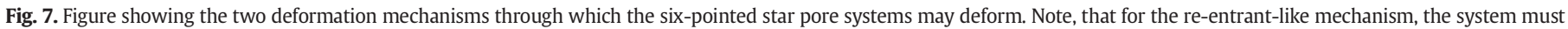
undergo shear deformations (see Fig. 8c), which are inhibited when the system is loaded using the boundary conditions of the tensile loading device.

shown in Fig. 2a(iii). This result indicates that another deformation appears to come into play at this stage, with this mechanism being similar to that observed for conventional hexagonal honeycombs, hence the large positive Poisson's ratio. Here it is also worth noting that for all these star-perforated systems, the structures with the lowest pore-tooverall-surface-area ratio possess the lowest Young's moduli and

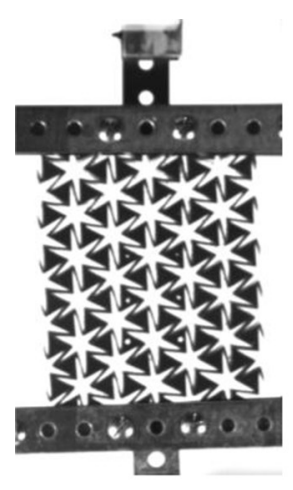

a)

$$
\varepsilon_{y}=0
$$

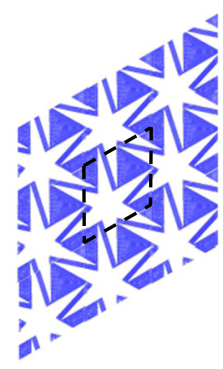

c)
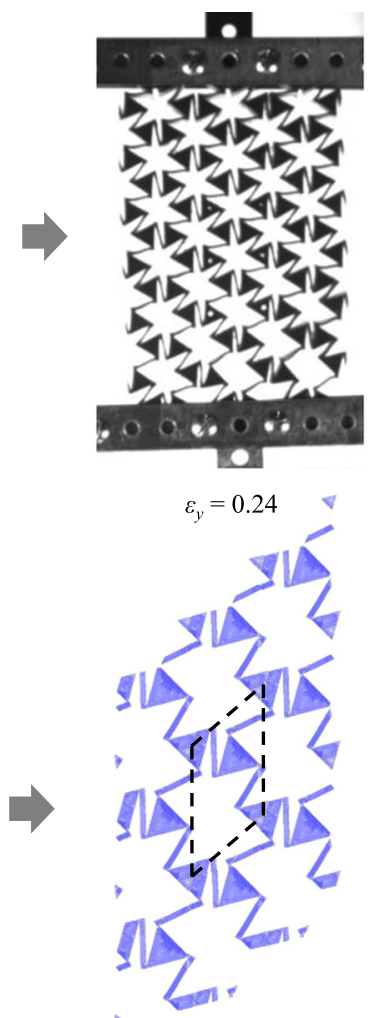

1) $\varepsilon_{y}=0.24$

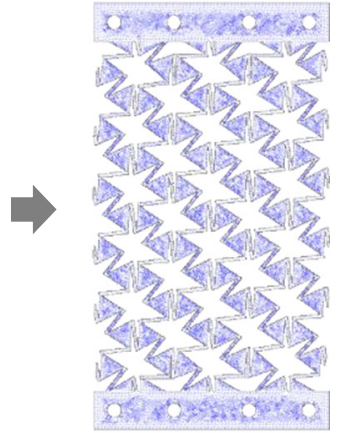

b)

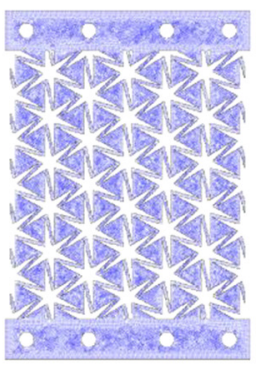

$\varepsilon_{y}=0$ $\varepsilon_{y}=0.24$

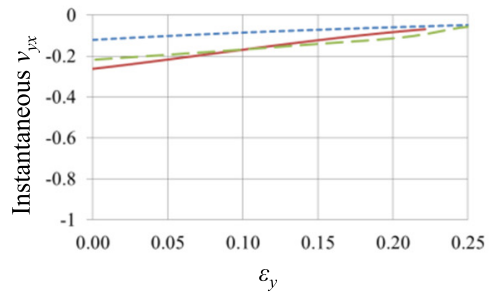

Experimental - - - FEA (Exp BC)

FEA (PBC)

d)

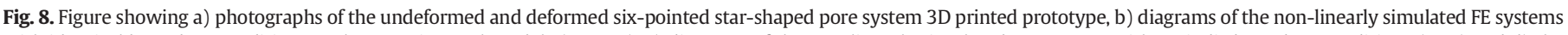

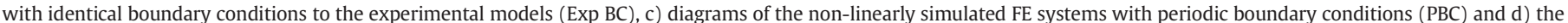

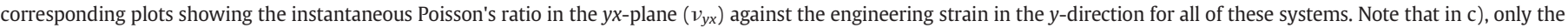

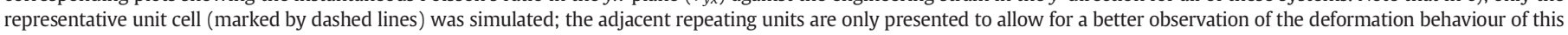
system. 
Poisson's ratio (see Fig. 2). This is because these systems typically possess a large $h / b$ ratio, which means that they have very small aperture angles for the pores. Therefore these systems are the farthest away from their fully-opened state, and are thus expected to have the greatest potential for expansion upon stretching.

The significance of the work presented in this paper goes beyond the results obtained here. For instance, these systems, despite the similarity in shape and deformation mechanism to typical circular node chiral honeycombs, are expected to exhibit superior performance upon tensile loading compared to these systems. This prediction is primarily based on the fact that the geometry of these systems is at a state that is farther away from the fully opened conformation of the chiral honeycomb mechanism than the respective typical circular node honeycombs, where the ligaments are tangentially oriented with respect to the center of the chiral node. This characteristic is envisaged to provide them with the added advantage of further scope for expansion upon the application of tensile loading while retaining their auxeticity/zero Poisson's ratio. In addition, although one may note that all the metamaterial geometries presented here exhibit a Poisson's ratio which stands within the rather limited confines of the range between 0 and -1 (i.e. the full range for isotropic auxeticity), this property only arises due to the highly regular and symmetric nature of the perforation shapes and arrangement being used. However, by reducing the symmetry of the star-shaped perforation, one may obtain chiral honeycomb-like systems with irregular polygonal shapes such as rectangles instead of squares, which would be expected to significantly affect the Poisson's ratio of the system. Indeed, previous studies on chiral honeycombs have shown that by changing the symmetry of the unit cell (which invariably happens if one changes the symmetry of the chiral node or uses more than one ligament length), one may obtain a vast range of negative Poisson's ratios [30,36,44]; thus it is not far-fetched to predict that a similar effect would probably also be observed in these systems. This concept could also potentially be extended to include 3D variants of these systems where 3D equivalents of the star-shaped perforations presented here could be used to create metamaterials capable of exhibiting 3D auxetic behaviour.

The versatility of these metamaterials is well suited for a variety of potential applications. In skin grafts, for example, star-perforations similar to those proposed here could be introduced into the graft in order to significantly extend its expandability in multiple dimensions. This would allow for a smaller skin graft to be used to cover up a much larger surface area. The negative Poisson's ratio properties of these systems also make them ideally suited for other niche applications in the biomedical field such as stenting, where geometries which show large expandability and synclastic curvature are required. In addition, in view of their characteristic geometries, these metamaterials could also potentially be suitable for a number of applications for which circular chiral honeycombs have been proposed such as morphing wing [45] and satellite antenna components [46].

\section{Conclusions}

In conclusion, we present a novel class of mechanical metamaterials with star-shaped pores which have the potential to exhibit zero and negative Poisson's ratios. This effect has been confirmed using both finite element simulations and substantiated by experimental evidence gathered from the testing of 3D printed prototypes. It was shown that the mechanical properties of these systems may be tailored simply by changing the star-perforation geometric parameters (i.e. size, openness and number of points of the star perforation) as well as the distance between perforations. A detailed analysis of the deformation behaviour and influence of boundary/ edge effects on the mechanical properties of these systems was also presented, as well as a study on the high strain properties of these systems and the range over which their negative Poisson's ratios may be retained. In addition, we have also shown how the systems proposed here may be used as a blueprint for the development of other related star-shaped pore systems with the potential to exhibit an even wider range of negative Poisson's ratios. Given the versatility and general applicability of the proposed structural systems, it is hoped that the work conducted here will open up new avenues to instigate design and 3D manufacture of auxetic mechanical metamaterials.

The raw data required to reproduce these findings are available to download from https://zenodo.org/record/1164866\#.WnSErKinGUk. Supplementary data to this article can be found online at https://doi. org/10.1016/j.matdes.2018.02.051.

\section{Acknowledgements}

LM and JCT thank The Royal Society for the award of the "Commonwealth Science Conference Follow-on Grants (2015 Round)" for financial support (CS150045).

\section{References}

[1] J.N. Grima, R. Caruana-Gauci, Nat. Mater. 11 (2012) 565-566.

[2] K.E. Evans, M.A. Nkansah, I.J. Hutchinson, S.C. Rogers, Nature 253 (1991) 124.

[3] A. Alderson, K.E. Evans, Phys. Rev. Lett. 89 (2002), 225503.

[4] M.R. Ryder, B. Civalleri, J.C. Tan, Phys. Chem. Chem. Phys. 18 (2016) 9079-9087.

[5] J.N. Grima, S. Winczewski, L. Mizzi, M.C. Grech, R. Cauchi, R. Gatt, D. Attard, K.W. Wojciechowski, J. Rybicki, Adv. Mater. 27 (2015) 1455-1459.

[6] L.J. Hall, V.R. Coluci, D.S. Galvao, M.E. Kozlov, M. Zhang, S.O. Dantas, R.H. Baughman, Science 320 (2008) 504-507.

[7] R.S. Lakes, Science 235 (1987) 1038-1040.

[8] M. Bianchi, F. Scarpa, M. Banse, C.W. Smith, Acta Mater. 59 (2011) 686-691.

[9] S. Neelakantan, J.C. Tan, A.E. Markaki, Scr. Mater. 106 (2015) 30-33.

[10] S. Dey, D.M. Agra-Kooijman, W. Ren, P.J. McMullan, A.C. Griffin, S. Kumar, Crystals 3 (2013) 363-390.

[11] R. Gatt, M. Vella-Wood, A. Gatt, F. Zarb, C. Formosa, K.M. Azzopardi, A. Casha, T.P. Agius, P. Schembri-Wismayer, L. Attard, N. Chockalingam, J.N. Grima, Acta Biomater. 24 (2015) 201-208.

[12] K. Bertoldi, P.M. Reis, S. Willshaw, T. Mullin, Adv. Mater. 21 (2009) 361-366.

[13] F. Scarpa, L.G. Ciffo, Y.R. Yates, Smart Mater. Struct. 13 (2004) 49-56.

[14] R.S. Lakes, K.E. Elms, J. Compos. Mater. 27 (1993) 1193-1202.

[15] M. Bianchi, F. Scarpa, Smart Mater. Struct. 22 (2013), 084010.

[16] Y. Chen, T. Li, F. Scarpa, L. Wang, Phys. Rev. Appl. 7 (2017), 024012.

[17] S.K. Bhullar, A.T.M. Hewage, A. Alderson, K.L. Alderson, M.B.G. Jun, Adv. Mater. 2 (2013) 42-47.

[18] M.N. Ali, I.U. Rehman, J. Mater. Sci. Mater. Med. 22 (2011) 2573-2582.

[19] L. Mizzi, D. Attard, A. Casha, J.N. Grima, R. Gatt, Phys. Status Solidi B 251 (2014) 328-337.

[20] Y. Cho, J.H. Shin, A. Costa, T.A. Kim, V. Kunin, J. Li, S.Y. Lee, S. Yang, H.N. Han, I.S. Choi, D.J. Srolovitz, PNAS 111 (2014) 17390-17395.

[21] R. Gatt, L. Mizzi, J.I. Azzopardi, K.M. Azzopardi, D. Attard, A. Casha, J. Briffa, J.N. Grima, Sci. Rep. 5 (2015) 8395.

[22] S. Babaee, J. Shim, J.C. Weaver, E.R. Chen, N. Patel, K. Bertoldi, Adv. Mater. 25 (2013) 5044-5049.

[23] H.H. Huang, B.L. Wong, Y.C. Chou, Phys. Status Solidi B 253 (2016) 1557-1564.

[24] T. Li, X. Hu, Y. Chen, L. Wang, Sci. Rep. 7 (2017) 8949.

[25] T. Li, L. Wang, Compos. Struct. 175 (2017) 46-57.

[26] T. Buckmann, N. Stenger, M. Kadic, J. Kaschke, A. Frolich, T. Kennerknecht, C. Eberl, M. Thiel, M. Wegener, Adv. Mater. 24 (2012) 2710-2714.

[27] A. Airoldi, P. Bettini, M.F. Panichelli, G. Sala, Phys. Status Solidi B 252 (2015) 1446-1454.

[28] B. Florijin, C. Coulais, M. van Hecke, Phys. Rev. Lett. 113 (2014), 175503.

[29] M. Taylor, L. Francesconi, M. Gerendas, A. Shanian, C. Carson, K. Bertoldi, Adv. Mater. 26 (2014) 2365-2370.

[30] L. Mizzi, K.M. Azzopardi, D. Attard, J.N. Grima, R. Gatt, Phys. Status Solidi (RRL) 9 (2015) 425-430.

[31] G. Carta, M. Brun, A. Baldi, Mech. Mater. 97 (2016) 67-75.

[32] Z. Zguris, How the Mechanical Properties of Stereolithography 3D Prints Are Affected by UV Curing, Formlabs White Paper, https://formlabs.com/media/upload/ How-Mechanical-Properties-of-SLA-3D-Prints-Are-Affected-by-UV-Curing.pdf 2016.

[33] C.W. Smith, J. Wootton, K.E. Evans, Exp. Mech. 39 (1999) 356-362.

[34] D. Prall, R.S. Lakes, Int. J. Mech. Sci. 31 (1997) 305-307.

[35] A. Alderson, K.L. Alderson, D. Attard, K.E. Evans, R. Gatt, J.N. Grima, W. Miller, N. Ravirala, C.W. Smith, K.M. Zied, Compos. Sci. Technol. 70 (2010) 1042-1048.

[36] J.N. Grima, Ph.D. Thesis, University of Exeter, United Kingdom, 2000.

[37] G. Cicala, G. Recca, L. Oliveri, Y. Perikleous, F. Scarpa, C. Lira, A. Lorato, D.J. Grube, G. Ziegmann, Compos. Struct. 94 (2012) 3556-3562.

[38] A. Alderson, K.L. Alderson, G. Chirima, N. Ravirala, K.M. Zied, Compos. Sci. Technol. 70 (2010) 1034-1041.

[39] A. Bacigalupo, L. Gambarotta, Compos. Struct. 116 (2014) 461-476. 
[40] C.W. Smith, J.N. Grima, K.E. Evans, Acta Mater. 48 (2000) 4349.

[41] N. Gaspar, X.J. Ren, C.W. Smith, J.N. Grima, K.E. Evans, Acta Mater. 53 (2005) 2439.

[42] Y. Jiang, Y. Li, Adv. Eng. Mater. 19 (2017), 1600609.

[43] S. Timoshenko, J.N. Goodier, Theory of elasticity, Engineering Societies Monographs, 1951.
[44] R. Gatt, D. Attard, P.S. Farrugia, K.M. Azzopardi, L. Mizzi, J.P. Brincat, J.N. Grima, Phys. Status Solidi B 250 (2013) 2012-2019.

[45] A. Spadoni, M. Ruzzene, J. Intell. Mater. Syst. Struct. 18 (2007) 1067-1075.

[46] S. Jacobs, C. Coconnier, D. DiMaio, F. Scarpa, M. Toso, J. Martinez, Smart Mater. Struct. 21 (2012), 075013. 